à fait comme la pasteurisation haute et utilise le même matériel ; elle finit également comme celle-ci, si ce n'est qu'entre l'intervention du pasteurisateur et l'intervention du réfrigérant, aux moyens des mêmes appareils, on fait intervenir des chambres spéciales. Déjà, avant que ce mode de pasteurisation ait été élevé au grade d'un vrai système, pas mal de laitiers avaient trouvé avantageux de garder quelque temps le lait à la sortie du pasteurisateur avant de le refroidir. De là est venue tout d'abord l'indication remplie par le fabricant Gaulin de construire un récipient intermédiaire, qui se place entre le pasteurisateur et le réfrigérant. Ensuite, a pris origine de la même indication le procédé de pasteurisation qui dispose aujourd'hui d'un matériel spécial de chambreurs, munis d'agitateurs mécaniques et de soupapes automatiques livrant passage au lait des chambreurs au réfrigérạnt aussitôt accompli dans chacun d'eux la période de vingt ou vingt-cinq minutes, jugée utile pour que le lait tout en sortant du pasteurisateur à $63^{\circ}$ accuse un degré suffisant de pasteurisation. Dans la figure 17, qui représente l'un des pasteurisateurs de la Laiterie Centrale de Strasbourg, on peut suivre le ehemin que le lait fait depuis la sortie du pasteurisateur jusqu'à l'un des quatre chambreurs avec lequel le pasteurisateur est relié dans ce système de pasteurisation basse.

Dans les deux eas, il y aura toujours à déplorer la perte en gaz earbonique et par évaporation que le lait subit étant traité en cireuit ouvert. L'épaisse buée que l'on remarque à l'arrivée du lait chaud sur le réfrigérant doit attirer l'attention du profane sur cette perte.

(A suisre.)

\title{
ÉTUDE SUR LA VITESSE DE VARIATION DANS LA COMPOSITION DU COLOSTRUM DE VACHE (1)
}

\author{
Par O.-R. OVERMAN et F.-P. SANMANN \\ de la Section d'Economie laitière \\ Université de l'Illinois, Urbana (Illinois).
}

Il existe déjà une documentation riche et variée sur la question du colostrum des diverses espèces de mammifères. En général, les renseignements donnés montrent que le colostrum diffère nettement du lait sécrété alors que la période de lactation dure depuis un cer-

(1) Une partie des renseignements utilisés dans cet artiele, proviennent d'une thèse soumise avee d'autres par F.-P. SAnmann, à la Faculté de l'Ecole supérieure de 1'Université de l'Illinois, en vue de l'obtention du titre de professeur d'économie laitière. Un exemplaire de la thèse originale existe à la Bibliothèque de l'Université de I'Illinois. 
tain temps. Le passage du colostrum au lait normal ne demandent ordinairement que quelques jours. Ensuite, les changements montrés par le " lait normal " sont moins prononés.

Le tableau I est le résumé de renseignements divers indiqués pà vingt-cinq expéximentateurs pour montrer les variations dans la composition du colostrum pendant les quatre premières traites après le vêlage. Les chiffres de ce tableau ne sont pas complets, et ne comprennent pas, dans tous les cas, les valeurs extrêmes des différents constituants, ear il a été impossible, dans beaueoup de eas, de grouper les renseignements obtenus d'une manière certaine dans les différentes traites. Kirch ner [15] indique que le poids spécifique peut atteindre et même dépasser 1:090. Aцвкеснт [24] signale également un poids spécifique de 1.090 pour le colostrum. Siegrield [5] signale qu'un échantillon prélevé deux jours après le vêlage, contenait $12 \%$ de matière grasse. ENGEL et DenNeMARK [16] indiquent avoir encore trouvé $12 \%$ de matière grasse le quatrième jour apvès le vêlage.

Comme les chiffres pour l'aeidité titrable ont été pris dans des publieations européennes, l'acidité est indiquée en " degrés ".

Les valeurs du poids spéeifique n'ont pas été indiquées dans tous les ceas, de sorte qu'il a été impossible de recalculer ces valeurs pour le pourcentage de l'acidité titrable. Enger et Dennemark [16] signalent un échantillon qui, le second jour après le vêlage, avait $50^{\circ} 05$ d'acidité titrable.

La plupart des chiffres employés pour la confection de ces tableaux ont été pris dans des publications plutôt anciènnes. Les méthodes actuelles d'analyse chimique et de meilleurs procédés d'expérimentation donneraient des résultats quelque peu différents, mais il semble raisonnable d'admettre que, dans l'ensemble, ces renseignements sont exacts.

On verra qu'il y a tendance vers une diminution rapide de traite en traite du pourcentage de l'extrait sec, de l'azote total, des cendres, une diminution de l'acidité et du poids spécifique, une augmentation du pourcentage de suore de lait et des variations plutôt importantes du pourcentage de la matière grasse. Les variations des différents constituants deviennent moins marquées au fur et à mesure qu'on s'éloigne de l'époque du vêlage.

La présente étude a eu pour but d'étudier plus complètement la vitesse de variation dans la eomposition du colostrum de vache, ainsi que l'effet de la fréquence des traites sur cette vitesse de variation.

Plan du trayall. - Pour cette étude, les vaches no 263 et $n^{\circ} 74$ du troupeau de l'Université de l'Illinois furent utilisées. La vache $n^{\circ} 263$ était une vache Holstein enregistrée, nêe le 12 déeembre 1917, 
et prête pour sa troisième période de lactation le 28 octobre 1922 . La dernière traite de sa précédente période de lactation avait été le 18 août 1922 . Elle avait été mise en état pour une production maxima, et pendant cette période de lactation, on la soumit à l'essai officiel tel qu'il est prescrit par l'Association Américaine des Eleveurs de la race Holstein-Frise.

La vache no 74 était une vache Ayrshire enregistrée, née le 17 mai 1906 , et prête pour sa quatorzième période de lactation le 3 décembre 1922. La dernière traite de sa précédente période de lactation avait été le 28 septembre 1922 . Elle était prête pour sa quinzième période de lactation le 3 mars 1924. Sa précédente dernière traite avait été le 16 septembre 1923.

La vache $n^{\circ} 263$ fut traite à fond et un échantillon prélevé aussitôt que possible après le vêlage, le 28 octobre 1922. Ensuite, elle fut traite à fond et des échantillons prélevés ehaque trois heures jusqu'à ce que cinq échantillons aient été obtenus. Par la suite, elle fut traite régulièrement quatre fois par jour et un échantillon prélevé sur chaque traite jusqu'à la fin du septième jour après le vêlage.

La vache $n^{0} 74$ fut traite à fond aussitôt que possible après le vêlage, le 3 décembre 1922, et le lait des moitiés gauche et droite du pis fut prélevé et échantillonné à part. Ensuite, la moitié gauche du pis fut traite à fond et échantillonnée deux fois par jour, tandis que la moitié droite l'était quatre fois par jour, jusqu'à la fin du septième jour après le vêlage. Chaque traite de la moitié droite du pis fut échantillonnée pendant les quatre premiers jours de la période de lactation. Le cinquième jour, un échantillon mélangé fut fait des première et deuxième traites, et un autre échantillon mélangé des troisième et quatrième traites. Les sixième et septième jours, des échantillons mélangés furent faits des quatre traites de chaque jour. Chaque traite de la moitié gauche du pis fut échantillonnée pendant les einq premiers jours de la période de lactation. Des échantillons mélangés furent faits des deux traites de chacun des sixième et septième jours, après vêlage. Pendant les sept premiers jours de sa quinzième période de lactation, commençant le 'le 3 mars 1924, l'expérience fut répétée avec la vache no 74, avec cctte différence que la moitié gauche du pis fut traite quatre fois par jour, et la moitié droite, deux fois par jour.

Tous les échantillons prélevés pour cette étude furent additionnés de formol employé à peu près dans la proportion recommandée par Palmer [28]. Ils furent conservés dans des bocaux à fruits en verre d'un demi-gallon (2 litres 27) avec joints en caoutchouc et couvercles de verre, et mis en glacière à environ $1^{\circ}$ à $3^{\circ} \mathrm{C}$. Occasionnellement, la température descendit au-dessous de zéro, de sorte que quelquesuns des échantillons furent gelés pendant la conservation. 
Dans tous les échantillons, on détermina : l'extrait sec, les cendres, l'azote total, la matière grasse, le lactose, l'acidité titrable, la densité et le point de congélation.

Ces études furent continuées tant avec la vache $n^{0} 263$ qu'avec la vache $n^{0} 74$, jusqu'à la fin de leurs périodes de lactation, mais les résultats ne seront pas consignés dans le présent article.

MÉTHOde de LABORATOIRE. - L'extrait sec fut déterminé par la méthode officielle.

Les cendres furent déterminées par la méthode officielle, avec cette différence que des échantillons de 10 gr., au lieu de 20 gr., furent utilisés, et la quantité d'acide azotique diminuée en conséquence. Ces déterminations furent faites dans des capsules de platine, à l'exception de quelques cas où on employa des capsules en silice fondue.

La matière grasse fut déterminée par la méthode officielle RoESEGotrlieb, avec cette différence que des échantillons d'environ $5 \mathrm{gr}$., au lieu de $10 \mathrm{gr}$., furent utilisés, et les quantités de réactifs réduites en conséquence. Dans le cas des six premiers échantillons de la vache $\mathrm{n}^{0} 74$, pendant sa quatorzième période de lactation, on a trouvé nécessaire de diluer les échantillons avee des volumes égaux d'eau, et d'augmenter les proportions de réactifs employés.

L'azote total fut déterminé par la méthode officielle de KJELDAHL, en employant du mercure métallique. Comme révélateur, le rougeméthyle-paranitrophénol fut employé.

Les dosages du lactose furent effectués par la méthode optique officielle, en employant du nitrate de mercure comme agent de précipitation. Pour les six premiers échantillons de la vache $\mathrm{n}^{0} \quad 7 \mathrm{t}_{\mathrm{t}}$ pendant sa quatorzième période de lactation, il fut trouvé nécessaire d'employer des quantités moitié moindres pour les déterminations et de corriger les lectures du saccharimètre en conséquence. Ces échantillons ayant des densités supérieures à celles du lait normal, furent pesés dans les flacons à précipité; tous les autres furent mesurés par le moyen d'une pipette à lactose spéciale [29]. Le pourcentage de lactose fut aussi obtenu en soustrayant la somme des pourcentages de cendres, matière grasse et azote total, du pourcentage d'extrait sec et les résultats furent comparés avec ceux obtenus par détermination directe.

L'acidité titrable fut déterminée en titrant des échantillons de 18 gr. avec une solution décinormale de soude, en employant la phénolphtaléine comme indicateur. Ce pourcentage d'acidité titrable fut calculé en acide lactique. Tous les titrages furent faits sur les échantillons avant le développement de l'acidité due à l'action bactérienne.

La densité fut déterminée à $15^{\circ} 5 \mathrm{C}$. avec une balance à densité 
du type à chaîne. On laissait les échantillons reposer quelques heures avant de procéder à cette détermination.

Le point de congélation fut déterminé dans chaque cas sur l'échantillon frais avant addition de l'antiseptique, et aussitôt que possible après la traite. Quand il n'était pas examiné immédiatement, l'échantillon était conservé en glacière pour éviter le développement de l'acidité. Les déterminations furent effectuées avee le cryoseope de Hortvet [26].

Toutes les déterminations furent faites en double, excepté la densité et le point de congélation. Quand les résultats des doubles déterminations n'étaient pas absolument d'aecord, on les répétait. Seules, les valeurs moyennes sont indiquées dans les tableaux.

Renseignements et piseussion. - Les résultats de eette étude sont donnés dans les tableaux II à X inclus.

Les renseignements pour la vache $n^{\circ} 263$ sont donnés dans le tableau II. On verra que des différences très marquées apparaissent dans la composition des échantillons obtenus de eette vaebe. Les changements les plus rapides se produisirent pendant les toutes premières traites, qui se succédèrent à courts intervalles.

L'extrait sec commenȩa avec le ehiffre élevé de $22,61 \%$, puis diminua rapidement pendant les toutes premières traites. Après cela, il y eut plus ou moins de fluctuations, avec une tendance générale à la baisse pendant la période d'études.

En général, les mêmes constatations furent faites pour les pourcentages de cendres, matière grasse, azote total, et acidité titrable. La tendance dans le cas de la matière grasse fut moins constante que dans les autres.

Le lactose commenęa avec le chiffre très bas de $2,02 \%$, par détermination directe; puis vint ensuite une diminution jusqu'à 1,62\% dans l'échantillon prélevé trois heures après. Ensuite, la tendance générale fut lentement en hausse, quoique marquée par beaucoup de fluctuations.

La densité diminua d'abord rapidement, puis continua avee une tendance lente à la baisse vers la fin de la période d'étude.

Le point de congélation commenȩa avec une valeur normale de 00554 C., puis baissa à 00584 C. à. la seconde traite, trois heures après. A l'exception du deuxième échantillon, an peut dire qu'aucun des échantillons ne donna de point de congélation différent de ceux qui pouvaient être attendus d'échantillons individuels de lait normal.

Les résultats indiqués dans le tableau I furent obtenus de chiffres provenant de vaches qui, pour la plupart, avaient été traites deux fois par jour. Il en résulte que les chiffres indiqués peuvent être considérés comme représentant le colostrum obtenu sur une période 


\section{TABLEAU I}

Composition du lait de vache.

Ce tableau a été obtenu en résumant les enseignements donnés par 25 expérimentateurs.

\begin{tabular}{|c|c|c|c|c|c|c|c|}
\hline & $\begin{array}{c}\text { Extrait } \\
\text { sec } \\
\%\end{array}$ & $\begin{array}{c}\text { Cendres } \\
\%\end{array}$ & $\begin{array}{c}\text { Matière } \\
\text { grasse } \\
\%\end{array}$ & $\begin{array}{c}\text { Azote } \\
\text { total } \\
\%\end{array}$ & $\begin{array}{c}\text { Lactose } \\
\%\end{array}$ & $\begin{array}{r}\text { Acidité } \\
\text { titrable } \\
\text { en degrés }\end{array}$ & Densité \\
\hline \multicolumn{8}{|l|}{ Premières traites : } \\
\hline Nombre d'analyses. & 66 & 58 & 73 & 54 & 53 & 52 & 59 \\
\hline Maximum. . . . & 38,40 & 2,31 . & 9,55 & 27,35 & 4,62 & 45,2 & 1,0830 \\
\hline Minimum. . . . & 13,72 & 0,68 & 0,15 & 4,80 & 0,00 & 11,5 & 1,0318 \\
\hline Moyenne. . & 24,55 & 1,33 & 3,89 & 16,76 & 2,50 & 18,4 & 1,0604 \\
\hline \multicolumn{8}{|l|}{ Secondes traites : } \\
\hline Nombre d'analyses. & 45 & 40 & 44 & 42 & 16 & 32 & 38 \\
\hline im. . & 31,11 & 1,37 & 9,0 & 19,47 & 4,7 & 36,8 & 1,0701 \\
\hline Minimum, & 11,83 & 0,6 & 0,5 & 5,01 & 2,37 & 7,9 & 1,0299 \\
\hline Moyenne... & 18,00 & 0,97 & 3,84 & 9,33 & 3,52 & 14,6 & 1,0437 \\
\hline \multicolumn{8}{|l|}{ Troisièmes traites : } \\
\hline Nombre d'analyses. & 17 & 16 & 15 & 17 & 12 & 13 & 13 \\
\hline Maximum. . & 27,62 & 1,2 & 5,1 & 17,90 & 4,44 & 19,8 & 1,0710 \\
\hline Minimum. & 12,89 & 0,67 & 0,5 & 4,85 & 2,7 & 11,0 & 1,0301 \\
\hline Moyenne... & 16,75 & 0,96 & 3,11 & 7,06 & 3,85 & 13,1 & 1,0376 \\
\hline \multicolumn{8}{|l|}{ Quatrièmes traites: } \\
\hline Nombre d'analyses. & 1 & . & 1 & 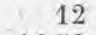 & 8 & 10 & \\
\hline $\mathrm{M}$ & 26,1 & 1,24 & 6,0 & 16,52 & 4,80 & 13,0 & 1,0625 \\
\hline $\mathrm{Mi}$ & 12,93 & 0,77 & 1,70 & 4,27 & 3,62 & 7,6 & 1,0300 \\
\hline Moyenne. . & 15,21 & 0,88 & 3,82 & 6,16 & 4,23 & 10,9 & 1,0372 \\
\hline
\end{tabular}

de deux jours. En comparant les résultats du tableau II avec ceux du tableau I, on constate qu'au bout de deux jours, la vache $n^{0} 263$ donnait du colostrum qui n'était pas partieulièrement différent de la moyenne, quoiqu'elle ait été traite dix fois depuis le vêlage. Dans ce cas, on voit que le fréquent enlèsement du colostrum n'a pas activé le passage de la composition pers celle du lait normal.

Avec la vache $n^{\circ} 74$, de façon à obtenir d'autres bases de comparaison, la moitié gauche du pis fut traite deux fois par jour, pendant que la moitié droite était traite quatre fois par jour, à intervalles égaux pendant les sept premiers jours de la quatorzième période de lactation. Puisque les deux moitiés du pis devaient normalement donner du lait de composition entièrement différente, on jugea convenable de répéter l'étude au début de la période suivante de lactation, mais en intervertissant cette fois les moitiés du pis.

Les chiffres obtenus pendant les deux périodes d'étude avęc la vache $n^{0} 74$ apparaissent dans les tableaux III à $X$ inclus. Pour plus de facilité, un tableau séparé a été préparé pour chaque détermination effectuée. Les heures d'échantillonnage et les quantités de lait obtenues dans les différentes traites sont seulement données dans le tableau III. Les échantillons indiqués dans les tableaux IV à X inclus sont, dans tous les cas, numérotés pour correspondre à ceux du tableau III. 
TABLEAU II

Vache $n^{\circ} 263$.

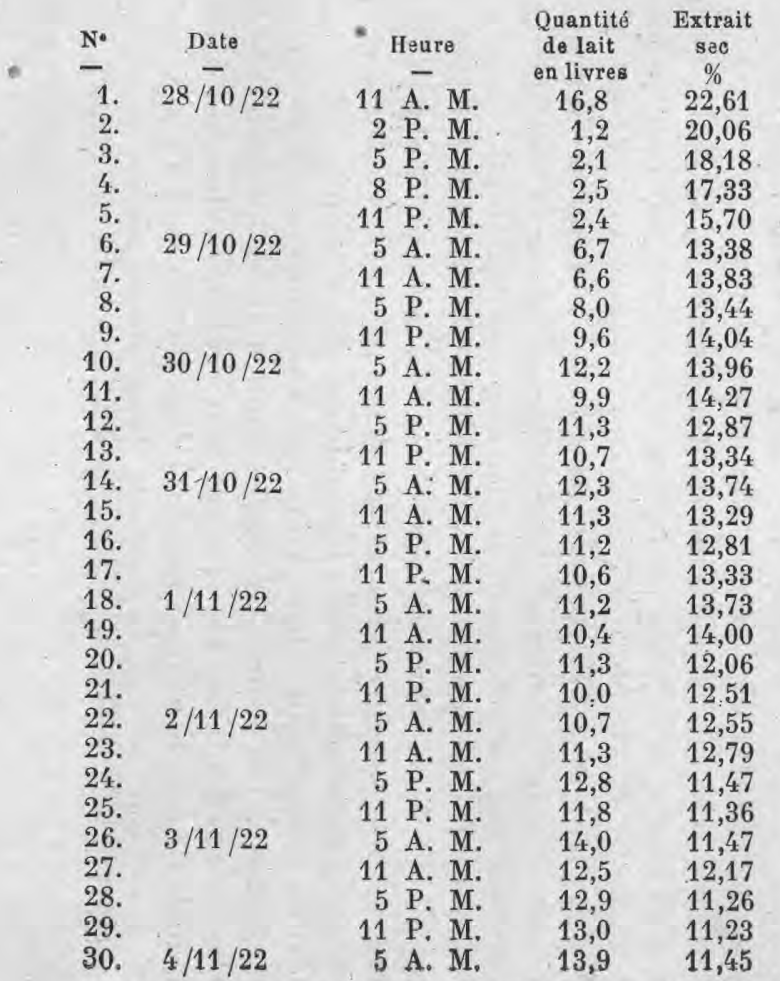

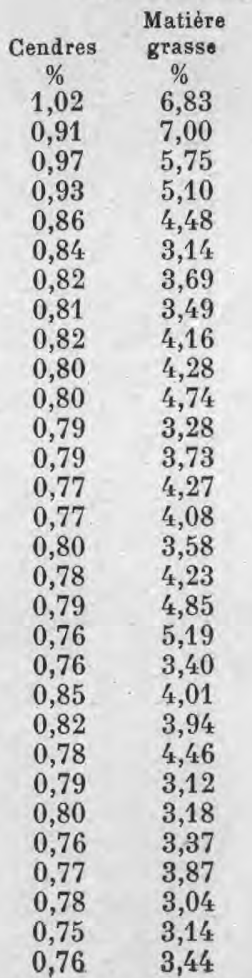

Azote Lactose par Lactose

total détermination par Acidité $\%$ directe $\%$ différence $\%$ titrable

$$
12,13
$$$$
\text { directe }
$$

9,90

8,58

7,78

6,55

5,78

5,39

5,23

5,15

5,01

4,83

4,85

4,74

4,58

4,42

-4,27

4,13

3,97

3,81

3,96

3,74

3,99

3,69

3,65

3,50

3,44

3,56

3,49

3,36

3,28$$
\begin{aligned}
& 2,02 \\
& 1,62
\end{aligned}
$$

1,62

$2,44 \quad 2,88$

$2,82 \quad 3,52$

$3,46 \quad 3,81$

$3,69 \quad 3,62$

$4,00 \quad 3,93$

3,92

3,99
$-3,94$

3,94

3,92

4,05

4,53

4,28

4,33

4,19

4,41

4,42
4,39

4,39

4,04
4,03

4,03
4,03

4,08

4,08

4,11

4,24

4,14

4,02
4,04

4,04
4,07

3,91

3,91

3,87

3,90

3,95

4,08

4,12

4,02

4,16

4,19

4,12

4,24

3,94

3,91

3,80

3,86

3,91

3,88

3,90

3,97

3,95

3,98

3,97

titrable
0,19

0,12

0,15

0,15

0,12

0,13

0,12

0,13

0,12

0,12

0,13

0,13

0,12

0,11

0,10

0,11

0,10

0,10

0,12

0,10

0,10

0,10

0,10

0,09

0,09

0,08

0,09

0,07
0,06

0,06

0,06

\begin{tabular}{cc} 
& \\
Densité & $\begin{array}{c}\text { Point de } \\
\text { congé- } \\
\text { lation }\end{array}$ \\
1,0501 & 0,554 \\
1,0404 & 0,584 \\
1,0406 & 0,573 \\
1,0392 & 0,575 \\
1,0371 & 0,564 \\
1,0357 & 0,558 \\
1,0338 & 0,557 \\
1,0338 & 0,553 \\
1,0343 & 0,558 \\
1,0335 & 0,546 \\
1,0337 & 0,565 \\
1,0353 & 0,559 \\
1,0351 & 0,561 \\
1,0331 & 0,560 \\
1,0331 & 0,555 \\
1,0341 & 0,567 \\
1,0329 & 0,562 \\
1,0319 & 0,560 \\
1,0323 & 0,557 \\
1,0315 & 0,556 \\
1,0316 & 0,560 \\
1,0303 & 0,552 \\
1,0310 & 0,551 \\
1,0314 & 0,546 \\
1,0313 & 0,546 \\
1,0296 & 0,548 \\
1,0309 & 0,552 \\
1,0306 & 0,551 \\
1,0303 & 0,544 \\
1,0293 & 0,548 \\
1 & \\
\hline &
\end{tabular}

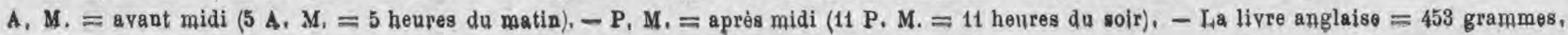




\section{TABLEAU III}

Vache $n^{0} 74 .-$ Heures d'échantillonnage, quantités de lait et extrait sec dans les échantillons.

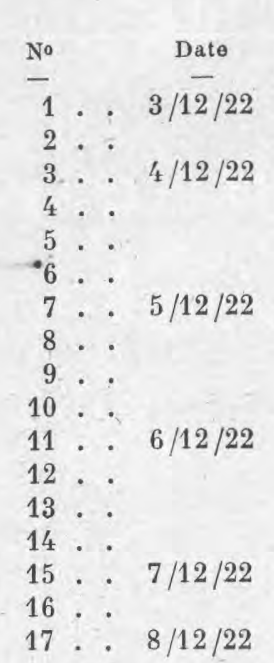

$$
\begin{aligned}
& 18 \ldots \\
& 19 \ldots 9 / 12 / 22 \\
& 20 \ldots 10 / 12 / 22
\end{aligned}
$$

\begin{tabular}{|c|c|c|c|c|}
\hline \multicolumn{2}{|c|}{$14^{\circ}$ lactation } & \multicolumn{2}{|c|}{ Extrait sec } & \\
\hline $\begin{array}{l}\text { Lait en livres } \\
\text { moitié } \\
\text { gauche }\end{array}$ & $\begin{array}{l}\text { Lait en livres } \\
\text { moitié } \\
\text { droite }\end{array}$ & $\begin{array}{c}\text { Moitié } \\
\text { gauche } \\
\%\end{array}$ & $\begin{array}{c}\text { Moitié } \\
\text { droite } \\
\%\end{array}$ & Date \\
\hline 2,8 & 2,5 & 27,70 & 30,29 & $3 / 3 / 24$ \\
\hline ") & 2,2 & ( ) & 26,11 & $4 / 3 / 24$ \\
\hline 4,0 & 2,8 & 24,64 & 21,02 & \\
\hline " & 1,8 & ") & 16,34 & \\
\hline 3,5 & 2,5 & 15,54 & 14,62 & \\
\hline ” & 3,2 & $n$ & 14,76 & $5 / 3 / 24$ \\
\hline 4,9 & 4,0 & 15,28 & 14,44 & \\
\hline$"$ & 3,3 & ") & 13,90 & \\
\hline 5,3 & 4,3 & 13,49 & 13,08 & \\
\hline$"$ & 4,2 & ) & 13,62 & $6 / 3 / 24$ \\
\hline 5,9 & 4,6 & 14,16 & 13,93 & \\
\hline ) & 4,6 & ") & 13,97 & \\
\hline 6,0 & 4,7 & 13,22 & 13,08 & \\
\hline$n$ & 4,5 & ) & 13,18 & $7 / 3 / 24$ \\
\hline 6,5 & 5,3 & 13,04 & 13,43 & \\
\hline D) & 4,4 & ) & 13,15 & \\
\hline 6,8 & 10,6 & 12,36 & 12,76 & $8 / 3 / 24$ \\
\hline 7,1 & 10,1 & 12,55 & 13,00 & $3 / 8 / 24$ \\
\hline 14,1 & 21,0 & 12,32 & 12,88 & $9 / 3 / 24$ \\
\hline 14,3 & 20,0 & 12,59 & 13,07 & $10 / 3 / 24$ \\
\hline
\end{tabular}

\begin{tabular}{|c|c|c|c|}
\hline \multicolumn{2}{|c|}{ 15. lactation } & \multicolumn{2}{|c|}{ Extrait sec } \\
\hline Lait en livres & Lait en livres & Moitié & Moitié \\
\hline moitié & moitié & gauche & droite \\
\hline gauche & droite & $\%$ & $\%$ \\
\hline 1,1 & 1,0 & 14,89 & 13,07 \\
\hline 0,9 & i) & 13,88 & ” \\
\hline 1,4 & 1,4 & 14,93 & 12,88 \\
\hline 2,2 & ") & 15,04 & $"$ \\
\hline 2,3 & 2,3 & 15,28 & 13,34 \\
\hline 2,6 & $n$ & 14,76 & $"$ \\
\hline 2,8 & 3,4 & 11,58 & 10,38 \\
\hline 2,8 & $"$ & 12,19 & 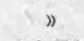 \\
\hline 1,9 & 2,0 & 12,47 & 11,78 \\
\hline 2,6 & $"$ & 13,38 & " \\
\hline 2,5 & 3,1 & 13,31 & 12,05 \\
\hline 2,1 & 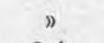 & 12,20 & $"$ \\
\hline 3,2 & 3,1 & 13,04 & 11,28 \\
\hline 2,7 & ) & 13,06 & " \\
\hline 3,0 & 3,0 & 13,44 & 12,34 \\
\hline 3,0 & $n$ & 13,08 & $n$ \\
\hline 5,8 & 3,5 & 13,40 & 11,14 \\
\hline 6,7 & 3,2 & 14,11 & 12,70 \\
\hline 13,1 & 6,5 & 13,52 & 11,82 \\
\hline 13,1 & 6,4 & 13,19 & $.11,90$ \\
\hline
\end{tabular}




\section{TABLEAU IV}

Vache $n^{0} 74$. Cendres.

\begin{tabular}{|c|c|c|c|c|c|}
\hline & & $14^{\circ} \mathrm{lac}$ & tation & $15^{\circ}$ lact & tation \\
\hline & & $\begin{array}{c}\text { Moitié gauche } \\
\%\end{array}$ & $\begin{array}{c}\text { Moitié droite } \\
\%\end{array}$ & $\begin{array}{c}\text { Moitié gauche } \\
\%\end{array}$ & $\begin{array}{c}\text { Moitié droite } \\
\quad \%\end{array}$ \\
\hline 1 & . . & 1,02 & 1,06 & 0,82 & 0,76 \\
\hline 2 & . . & D & 1,02 & 0,92 & 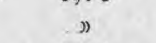 \\
\hline 3 & $\ldots$ & 1,01 & 0,92 & 0,94 & 0,90 \\
\hline 4 & $\ldots$ & ") & 0,87 & 0,91 & $"$ \\
\hline 5 & $\ldots$ & 0,91 & 0,84 & 0,90 & 0,94 \\
\hline 6 & $\ldots$ & $"$ & 0,82 & 0,85 & $"$ \\
\hline 7 & . . & 0,85 & 0,79 & 0.77 & 0.79 \\
\hline 8 & . . & ) & 0,77 & 0,78 & ” \\
\hline 9 . & . . & 0,81 & 0,77 & 0,85 & 0,88 \\
\hline 10. & $\therefore$ & w & 0,78 & 0,79 & 》) \\
\hline 11. & . . & 0,83 & 0,80 & 0,78 & 0,77 \\
\hline 12. & . . & ") & 0,80 & 0,76 & ") \\
\hline 13. & . . & 0,81 & 0,78 & 0,76 & 0,73 \\
\hline 14. & . . & 11 & 0,77 & 0,75 & $"$ \\
\hline 15. & $\therefore$ & 0,80 & 0,78 & 0,76 & 0,76 \\
\hline 16. & . . & 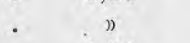 & 0,78 & 0,74 & $"$ \\
\hline 17. & . . & 0,80 & 0,78 & 0,78 & 0,76 \\
\hline 18 & . . & 0,79 & 0,79 & 0,76 & 0,73 \\
\hline 19 & . & 0,76 & 0,76 & 0,73 & 0,72 \\
\hline 20. & . . & . $\quad 0,78$ & 0,76 & 0,78 & 0,71 \\
\hline
\end{tabular}

TABLEAU V Matière grasse.

$14^{\mathrm{e}}$ lactation

15 lactation

Moitié gauche Moitié droite $\%$ $\%$

$1 \ldots 5,12$

6,08

2... "

4,86

3... 4,62

3,94

4 ...

2,95

$5 \ldots 2,61$

2,62

6 ...

3,82

7 . . . 3,87

3,86

8...

3,88

9... 3,36

3,24

$10 \ldots$...

3,85

$11 \ldots \quad 4,17$

4,03

12 ... "

4,38

13 ... 3,84

3,60

14 ...

3,74

15 . . 3,76

4,04

16 ... "

3,88

17 . . 3,37

3,52

$18 \ldots 3,42$

3,68 .

19 . . . 3,40

3,72

20 . . 3,85

3,94

\begin{tabular}{cc}
\hline Moitié gauche & Moitié droite \\
$\%$ & $\%$ \\
2,58 & 2,40 \\
2,38 & $" \prime$ \\
2,32 & 1,48 \\
2,76 & $\prime \prime$ \\
3,56 & 1,98 \\
3,62 & $\prime \prime$ \\
2,52 & 1,46 \\
3,04 & $" \prime$ \\
3,02 & 2,25 \\
4,02 & $" 1$ \\
4,03 & 3,41 \\
3,41 & $" \prime$ \\
3,90 & 3,20 \\
4,14 & $" \prime$ \\
4,48 & 3,84 \\
4,61 & $" \prime$ \\
4,61 & 3,64 \\
5,48 & 4,58 \\
4,80 & 4,04 \\
4,54 & 3,66
\end{tabular}


TABLEAU VI

Azote total.

$14^{\circ}$ lactation

Moitié gaache Moitié droite $\%$

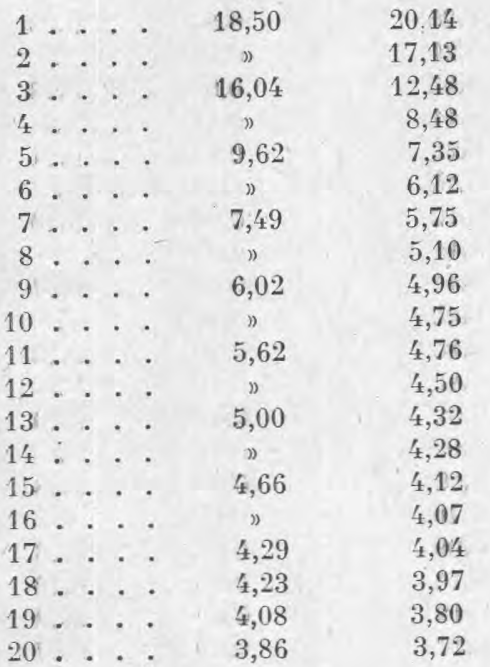

15e lactation

Moitié gauche Moitié droite

\begin{tabular}{rc}
\multicolumn{1}{c}{$\%$} & $\%$ \\
10,72 & 9,12 \\
9,62 & " \\
10,05 & 9,39 \\
9,22 & " \\
8,39 & 8,85 \\
7,22 & " \\
5,53 & 6,72 \\
5,32 & " \\
5,34 & 6,70 \\
5,22 & " \\
5,12 & 5,69 \\
4,66 & " \\
4,74 & 4,88 \\
4,52 & " \\
4,48 & 4,88 \\
4,05 & " \\
4,04 & 4,34 \\
4,15 & 4,42 \\
4,02 & 4,24 \\
3,79 & 3,86
\end{tabular}

\section{TABLEAU VII}

Lactose.

$14^{\circ}$ lactation

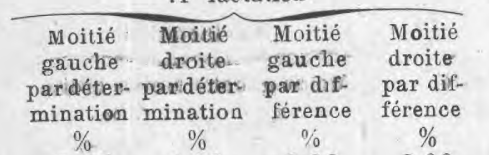

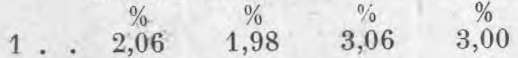

2. " 2,36 " 3,10

3. $\begin{array}{llll}2,45 & 3,79 & 2,97 & 3,68\end{array}$

4. " 3,59 " 4,04

5. $\begin{array}{llll}1,92 & 3,94 & 2,40 & 3,81\end{array}$

$\begin{array}{lllll}6 . .0 & 4,18 \text { " } & 4,00\end{array}$

7.. $2,80 \quad 4,25$

8 . $~ 4,33$

9. $3,24 \quad 4,28$

10 . " 4,47

11. $3.43 \quad 4,56$

12 . . $\quad 4,36$

13.. 3,4 $4,4,14$

14 . $>\quad 4,55$

15. . $3,78 \quad 4,66$

16 . \ 4,58

17. $3,99 \quad 4,71$

18 . $4,02 \quad 4,77$

19. . $3,99 \quad 4,76$

20 . $\quad 4,05 \quad 4,78$
3,07

1) 4,15

$3,30 \quad 4,11$

1) 4,24

$3,54 \quad 4,34$

1) 4,29

$3,57 \quad 4,38$

1). 4,39

$3,82 \quad 4,49$

i) 4,42

$3,90 \quad 4.42$

$4,11 \quad 4,56$

$4,08 \quad 4,60$

$4,10 \quad 4,65$ $15^{\mathrm{e}}$ lactation

$\begin{array}{cccc}\text { Moitié } & \text { Moitié } & \text { Moitié } & \text { Moitié } \\ \text { gauehe droite gauche } & \text { droite } \\ \text { par déter-par déter- } & \text { par dif- } & \text { par dif- }\end{array}$

mination mination férence férence

$\begin{array}{cccc}\% & \% & \% & \% \\ \text { négative } & 0,36 & 0,75 & 0,78 \\ 0,48 & \prime \prime & 0,96 & \prime \prime \\ 1,26 & 0,72 & 1,62 & 1,11 \\ 1,90 & \prime \prime & 2,15 & \prime \prime \\ 2,58 & 1,10 & 2,43 & 1,57 \\ 3,08 & \prime \prime & 3,07 & \prime \prime \\ 2,63 & 1,04 & 2,76 & 1,41 \\ 3,14 & \prime \prime & 3,06 & \prime \prime \\ 3,37 & 1,73 & 3,26 & 1,95 \\ 3,51 & \prime \prime & 3,35 & \prime \prime \\ 3,64 & 1,86 & 3,38 & 2,18 \\ 3,49 & \prime \prime & 3,37 & \prime \prime \\ 3,68 & 2,24 & 3,64 & 2,47 \\ 3,92 & \prime \prime & 3,65 & \prime \prime \\ 4,05 & 2,88 & 3,72 & 2,86 \\ 4,00 & \prime \prime & 3,68 & \prime \prime \\ 4,01 & 2,41 & 3,97 & 2,40 \\ 4,03 & 2,92 & 3,72 & 2,97 \\ 4,13 & 2,72 & 3,97 & 2,82 \\ 4,24 & 2,57 & 4,08 & 2,67\end{array}$




\section{TABLEAU VIII}

Acidité titrable.

14. lactation

Moitié gauche Moitié droite $\%$

1 .. 0,22

$\%$

2... ”

0,25

3 . . 0,18

0,21

4....

5 . . . 0,10

0,15

6 .

7 . . 0,13

$8 . . .0$

$9 \ldots 0,10$

$10 \ldots$

11 . . 0,09

12 .. 》

13 ... 0,08

14 .. 》

$15 . . \quad 0,09$

16 .. ग

17 . . 0,08

18 . . 0,07

19 .. $\quad 0,09$

20 ... 0,09
0,11

0,13

0,12

0,12

0,11

0,11

0,10

0,10

0,10

0,09

0,09

0,09

0,10

0,09

0,11

0,12

0,11 $15^{\circ}$ lactation

Moitié gauche Moitié droite

$\% \quad \%$

$0,17 \quad 0,08$

0,10 )

$0,09 \quad 0,08$

0,11 "

$0,11 \quad 0,11$

0,11 ,

$0,06 \quad 0,10$

0,08 n

$0,08 \quad 0,07$

0,12 "

$0,10 \quad 0,13$

0,10 "

$0,11 \quad 0,10$

0,11 "

$0,10 \quad 0,08$

$\begin{array}{ll}0,08 & \text { " } \\ 0,08 & 0,08\end{array}$

$0,09 \quad 0,10$

$0,09 \quad 0,08$

$0,09 \quad 0,07$

TABLEAU IX

Densité.

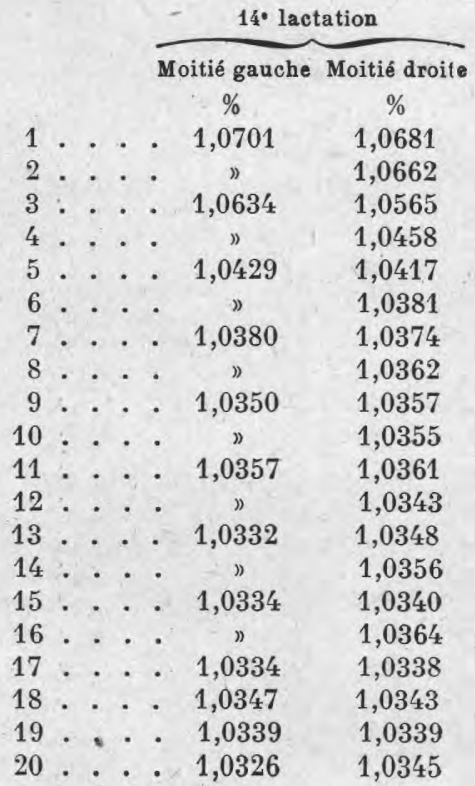

$15^{\mathrm{e}}$ lactation

Moitié gauche Moitié droite

$\begin{array}{cc}\% & \% \\ 1,0375 & 1,0336 \\ 1,0361 & \prime \prime \\ 1,0406 & 1,0366 \\ 1,0394 & \prime \prime \\ 1,0384 & 1,0372 \\ 1,0368 & \prime \prime \\ 1,0306 & 1,0302 \\ 1,0315 & " \\ 1,0329 & 1,0320 \\ 1,0323 & \prime \\ 1,0316 & 1,0290 \\ 1,0315 & \prime \\ 1,0319 & 1,0280 \\ 1,0316 & \prime \\ 1,0319 & 1,0296 . \\ 1,0300 & \prime \\ 1,0298 & 1,0252 \\ 1,0281 & 1,0282 \\ 1,0304 & 1,0254 \\ 1,0291 & 1,0257\end{array}$




\begin{tabular}{|c|c|c|c|c|c|c|c|c|}
\hline & $=$ & t & & $\begin{array}{r}\text { F } \\
14^{\mathrm{e}} \mathrm{las}\end{array}$ & $\begin{array}{l}\text { ABLEAU } \\
\text { int de congél } \\
\text { ation }\end{array}$ & $\begin{array}{l}\text { Xi11 } \\
\text { ation. }\end{array}$ & $15^{\circ} 1 \mathrm{a}$ & ctation \\
\hline & & & & $\begin{array}{c}\text { Moitié gauche } \\
\%\end{array}$ & $\begin{array}{c}\text { Moitié droite } \\
\%\end{array}$ & & $\begin{array}{c}\text { Moitié gauche } \\
\%\end{array}$ & $\begin{array}{c}\text { Moitié droite } \\
\%\end{array}$ \\
\hline 1 & . . & . & . & 0,586 & 0,586 & & 0,600 & 0,560 \\
\hline 2 & $\therefore$ & . & . & n) & 0,598 & & 0,605 & ) \\
\hline 3 & . . & . & . & 0,600 & 0,592 & & 0,588 & 0,577 \\
\hline 4 & . . & . & . & n & 0,587 & & 0,588 & 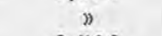 \\
\hline 5 & . . & . & . & 0,579 & 0,574 & & 0,560 & 0,590 \\
\hline 6 & $\therefore$ & . & . & ") & 0,577 & & 0,590 & $n$ \\
\hline 7 & . . & . & . & 0,579 & 0,575 & & 0,566 & 0,592 \\
\hline 8 & . . & . & . & ) & 0,582 & & 0,552 & ") \\
\hline 9 & . . & . & . & 0,573 & 0,574 & & 0,566 & 0,560 \\
\hline 10 & . . & . & : & D) & 0,581 & & 0,550 & \# \\
\hline 11 & . . & . & - & 0.577 & 0,589 & & 0,575 & 0,598 \\
\hline 15 & . . & . & . & D & 0,575 & & 0,569 & ") \\
\hline 13 & . . & . & . & 0,565 & 0,566 & & 0,549 & 0,564 \\
\hline 14 & . . & . & . & ) & 0,564 & & 0,542 & ” \\
\hline 15 & . . & . & . & 0,570 & 0,568 & & 0,577 & 0,570 \\
\hline 16 & . . & . & . & ) & 0,564 & & 0,567 & ) \\
\hline 17 & . . & . & 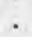 & 0,559 & 0,563 & & 0,562 & 0,572 \\
\hline 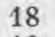 & . . & . & . & 0,576 & 0,582 & & 0,559 & 0,579 \\
\hline 1 & . . & . & . & 0,568 & 0,563 & & 0,556 & 0,563 \\
\hline 20 & . . & . & . & 0,574 & 0,570 & & 0,562 & 0,565 \\
\hline
\end{tabular}

Les résultats obtenus avec ehaque moitié du pis pendant chacune des périodes de lactation étudiées avec la vache $\mathbf{n}^{0} 74$, sont, dans les grandes lignes, tout à fait comparables à ceux obtenus avec la vache $n^{0}$ 263. Ni dans l'une, ni dans l'autre des périodes de lactation, les échantillons obtenus des moitiés séparées du pis ne furent semblables, mais les différences sont celles auxquelles on pouvait raisonnablement s'attendre même quand les deux moitiés ne sont. pas traitées différemment. Il apparaît que l'enlèvement du colostrum $d u$ pis à des intervalles plus fréquents n'influence pas la vitesse de sa variation de composition. Apparemment, la vitesse de variation de la composition est plus ou moins définie pour chaque individu. De plus fréquents enlèvements du colostrum déjà sécrété permettent seulement d'étudier les ehangements à un plus grand nombre de moments.

Il est possible, lorsqu'une moitié du pis de la vache est traite plus fréquemment que l'autre, qu'il y ait entre elles quelque relation de sympathie qui affecte la composition du lait par chacune. Il est admis cependant que ee facteur, s'il existe, n'est pas suffisamment prononcé pour annuler la comparaison.

La partie la plus intéressante de cette étude fut celle concernant le lactose. Crusius [20] avait indiqué qu'un échantillon pris d'une vache immédiatement après vêlage, ne contenait pas de lactose. Dans le tableau VII, le lactose du premier échantillon de la moitié gauche du pis de la vache $n^{0} 74$ dans sa quinzième période de lactation est donné comme " négatif ». La solution préparée de cet échan- 
tillon pour la détermination optique du lactose donna une légère rotation à gauche au lieu d'une rotation à droite à l'examen au saccharimètre. Cet échantillon réduisait très légèrement la liqueur de Fehling, la solution d'essai devenant d'une coloration verdâtre. Aucun précipité rougeâtre n'était visible à l'œil nu. Comme l'échantillon avait été conservé avec du formol, il est probable que toute la réduction était due à la trace d'aldéhyde présente. Des tentatives pour préparer un asazone avec de la phénylhydrazine furent infructueuses. D'autres essais ne furent pas possibles en raison de ce que l'échantillon était épuisé.

Dans cette étude, le lactose fut déterminé " par différence " en supplément de la déterminațion optique direete. En règle générale, on trouva que les différences entre les résultats pour le lactose « par différence » et par détermination directe furent plutôt importantes dans le cas des échantillons prélevés tout près du vêlage. Au fur et à mesure que les échantillons se rapprochaient du lait normal, les deux chiffres powr le lactose se rapprochaient. Il n'a pas été essayé dans cette étude d'expliquer cette différence.

Résumé. - La composition du colostrum de vache, telle qu'elle a été observée dans la présente étude, ehange rapidement après le vêlage. Dans le cas de la vache $n^{\circ} 263$, il ne semble pas que la traite complète à intervalles fréquents affecte la vitesse de variation dans la composition du colostrum. Dans le cas de la vache $\mathbf{n}^{0} 74$, il n'apparât pas que la traite d'une moitié du pis effeetuée plus fréquemment que celle de l'autre moitié, affecte la vitesse de variation dans la composition du colostrum, aussi bien dans l'une que dans l'autre moitié du pis. En apparence, la vitesse de variation dans la composition du colostrum de vache n'est pas nettement influencée par la fréquence des traites, mais dépend probablement de l'individualité de l'animal.

Un échantillon de la vache $\mathbf{n}^{\circ} 74$ ne contenait pas de laetose déterminable par les méthodes d'analyse employées.

\section{Bibliographie}

[1] W.-W. Сооке et J.-L. Hruss, Vt. Agr. Exp. Sta. Rpt., 111-113, 1891.

[2] F.-G. Deissmann, Chem. Zentr., 68, II, 1057, 1897.

[3] G. Srmox, Hoppe-Seyler's Z. physiol. Chem., XXXIII, 466-541, 1901.

[4] J.-L. Hinıs, Vt. Agr. Exp. Sta. Rpt., 104-108, 1891.

[5] M. Siegreld, Molkerei Ztg. (Hildesheim), XXII, 1293, 1908. Cf. Milchwirtschaft. Zentr., V, 37-38, 1909.

[6] H. Tuemann, Z. physiel. Chem., XXV, 363-392, 1898.

[7] C-H. Eckles et L.-S. Palmer, J. Biol. Chem., XXVII, 313-326, 1916.

[8] E. Winterstein et E. Strickler, Hoppe-Seyler's Z. physiol: Chem., XLVII, 58-82, 1906.

[9] W.-F. Sutherst, Chem. Nesss, LXXXVT, 1-2, 1902.

[10] E. Wener, Milchwirtschait. Zentr., VI, 433-449, 481-492, 543-563, 1910 (contient une bibliographie de 164 travaux). 
[11] W. Fleischmand, Lehrbuch der Milchwirtschaft, p. 40, 1893.

[12] Eugling, Bericht u. d. Thatigk. d. Iandw chem. Vers. Stat. d. Landes Vorarlberg, p. 33-41, 1878. Cf. n ${ }^{\circ} 11$, ci-dessus, p. 41.

[13] A. Burr, F.-M. Berberich et A. Berg, Chem. Zeit, XXXViI, 69-71, 1913.

[14] C. Konnautr, Z. Nahrungsmittelunter. Hyg., III, 5 janv. Cf. Chem. Zentr., 60, I, 294, 1889.

[15] Kirchner, Handbuch der Milchsirtschaft, p. 27, 45-50. Cf. no 10, ci-dessus, p. 559.

[16] St. Enget et L. Dennemark, Hoppe-Seyler's Z. physiol. Chem., LXXVI, 148-158, 1912.

[17] T. Henkex, Milchwirtschaft. Zentr., III, 340-369, 378-405, 1907.

18] Sebelien, Chem. Zeit., XVI, 597, 1892.

[19] L. Vaudin, Bull. Soc. Chim., XI-XII, 623-625, 1894. Cf. Exp. Sta. Record, VI, 335, 1894-1895.

[2]0 F. Crusius, $J$. prakt. Chem., I, 68, 1856.

[21] V. Howdet, Ann. Inst. Pasteur, VIII, 506-513, 1894. C. Chem. Zentr., 65, II, 525, 1894.

[22] Sato, Trans. Sapporo. Nat. Hist. Soc., V, 96-110, 1914. Cf. Chem. Abs., IX, 669, 1915.

[23] Kruger, Molkerei Ztg., VI, no 16, 1892. Cf. Chem. Zentr., 63, II, 1023-1024, 1892.

[24] Azвreent, Wochenschrift f. Tierheilkunde u. Viehzucht, L, 601, 1906. Cf. $\mathrm{n}^{0} 10$, ci-dessus, p. 561 .

[25] C.-J. Koning, Milchwirtschaft. Zentr., V, 101-113, 156-180, 1909.

[26] J. Hontvet, J. Ind. Eng. Chem., XIII, 198-208, 1921.

[27] Association of Official Agricultural Chemists. Official and Tentative Methods of Analysis, 1920.

[28] L.-S. Palmer, Mo. Agr. Exp. Sta. Ries. Bul.. XXXIV, 29, 1919.

[29] E.-G. Maнin, J. Ind. Eng. Chem., XV, 943, 1923.

\section{IMODIFICATION ET SIMPLIFICATION DU PROCÉDÉ PROPOSÉ PAR M. LE D ${ }^{r}$ HÖYBERG POUR LA DÉTERMINATION DE LA MATIÈRE GRASSE DANS LE LAIT ET LA CRĖME}

Par Bernhard SPUR

Laboratoire biochimique de l'Institat Polytechnique Royal de Copenhague (Directeur : Professeur OrLa-JRessen).

$$
\text { - SUITE BT FIN - }
$$

Les déterminations de la crème sont partout comparées avec celles effectuées suivant le procédé Röse-Gottlieb, parce que celui de Gerber ne nous paraît pas tout à fait sûr en ce qui la concerne. Parmi les déterminations de crème, on en trouvera aussi sur des crèmes pour l'exportation (crème homogénéisée et stérilisêe). L'échelle des butyromètres pour lait allant jusqu'à $8 \%$, et celle des butyromètres pour crème s'étendant même de 0 jusqu'à $60 \%$, il est démontré dans les tableaux que les deux sortes de déterminations passent, dans une certaine mesure, l'une dans le territoire de l'autre, c'est-à-dire qu'on peut déterminer la 